\title{
Instrument Modeling Concepts for Tradespace Analysis of Satellite Constellations
}

\author{
Sreeja Nag ${ }^{1,2}$, Vinay Ravindra ${ }^{1,2}$, Jacqueline Le Moigne ${ }^{1}$ \\ ${ }^{1}$ NASA Goddard Space Flight Center (Greenbelt, MD, U.S.A.) \\ ${ }^{2}$ Bay Area Environmental Research Institute (Moffett Field, CA, U.S.A.) \\ Sreeja.Nag@nasa.gov
}

\begin{abstract}
Constellations are gaining popularity in government and commercial space-based missions for Earth Observation (EO) due to their risk tolerance and ability to improve observation sampling in space and time. NASA Goddard Space Flight Center (GSFC) is developing a pre-Phase A tool called Tradespace Analysis Tool for Constellations (TAT-C) to initiate constellation mission design. The tool will allow users to explore the tradespace between various performance, cost and risk metrics (as a function of their science mission) and select Pareto optimal architectures that meet their requirements. This paper will describe the concept of modeling the primary science instruments within TAT-C, using a radar as an example, but extendable to imagers, occulters and lidars. The modularity of TAT-C's software architecture allows for crisply defining the interface between TAT-C's user defined or internal variables and the payload variables. The described module will inform TAT-C users of payload-dependent performance differences among thousands of constellation architectures (e.g. revisit time of the sensor swath, differential signal to noise ratio (SNR), spatial resolution of measurements) and allow them to pick an appropriate constellation architecture for detailed development. The module may also inform operational decisions of satellite modes, based on ground optimization or onboard autonomy.
\end{abstract}

Keywords-Satellites, Distributed sensors, Systems Engineering, Space mission design, Remote sensing.

\section{INTRODUCTION}

Distributed sensors in space, if coordinated and calibrated effectively, are capable of becoming a very powerful and scalable infrastructure in Earth remote sensing. NASA GSFC is leading the development of Tradespace Analysis Toolkit for Constellations (TAT-C) [1], which will allow scientists to explore constellation mission architectures, that minimize cost and maximize performance for pre-defined science goals, and will be aided by knowledge databases and machine learning. In a prior publications[2], [3], we described the executive driver of TAT-C, which ingests user inputs, enumerates and searches the all possible architectures, calls all the other modules and arranges the results of each architecture neatly into a file tree. The orbit, coverage, data reduction and metric computation modules were validated[2] against AGI's Systems Tool Kit (STK) via two use cases. This paper will conceptualize the observational instrument module within TAT-C, to support a variety of remote sensing applications. To our knowledge, the new contributions of this work are: (1) conceptualizing instrument trades within a tradespace search and evaluation tool for Earth observing constellations, with more design variables and performance outputs than published in academic literature before; (2) identifying the key dependencies between instrument and constellation design variables so that the most significant trade are presented to the user; and (3) building a framework for future adaptive remote sensing where instrument modes can change over time.

\section{InStRumENT Modeling Methodology}

The information flow within TAT-C for performance metric computation, for a tradespace of constellation architectures, is shown in Ref [2, Fig. 1]. We expand the capability of the TAT$\mathrm{C}$ tool to allow for simulation of instrument operations within the constellation, taking into account the type of instrument (imagers, radar, etc), and the instrument specifications and operational parameters. Fig. 1 shows the conceptual framework of the instrument simulation module. It is implemented essentially as an iterative block that is called by the Distributed Space Mission (DSM) Evaluator, which computes the performance of any constellation architecture generated within TAT-C. The instrument module uses results from the orbit propagator and coverage calculator, as published within the user's file tree [3]. The decoupling and placement of the instrument simulation module after the orbit propagation unit is possible because we assume there is no feedback to the satellite orbit propagator from the instrument module. This feedforward design is a reasonable assumption, since typically, orbit maneuvers are performed by a ground station operator and not by any autonomous feedback involving the instrument states.

There are three main functions (submodules) of the instrument module described in detail in following paragraphs.

\section{A. Instrument specifications search-space generation submodule}

This submodule generates the instrument architectures with different specifications, within constraints as defined by the user. Any given constellation can have many options of instruments, as picked by the selection switch in Fig. 1. A simple approach of uniform discretization is used for generation of the search space of the instrument specifications. The specifications are classified into two-types:

1) Non-operational specifications:

These are non-varying, hard specifications of the instrument, such as the physical area of antenna in a Synthetic Aperture Radar (SAR) instrument. 


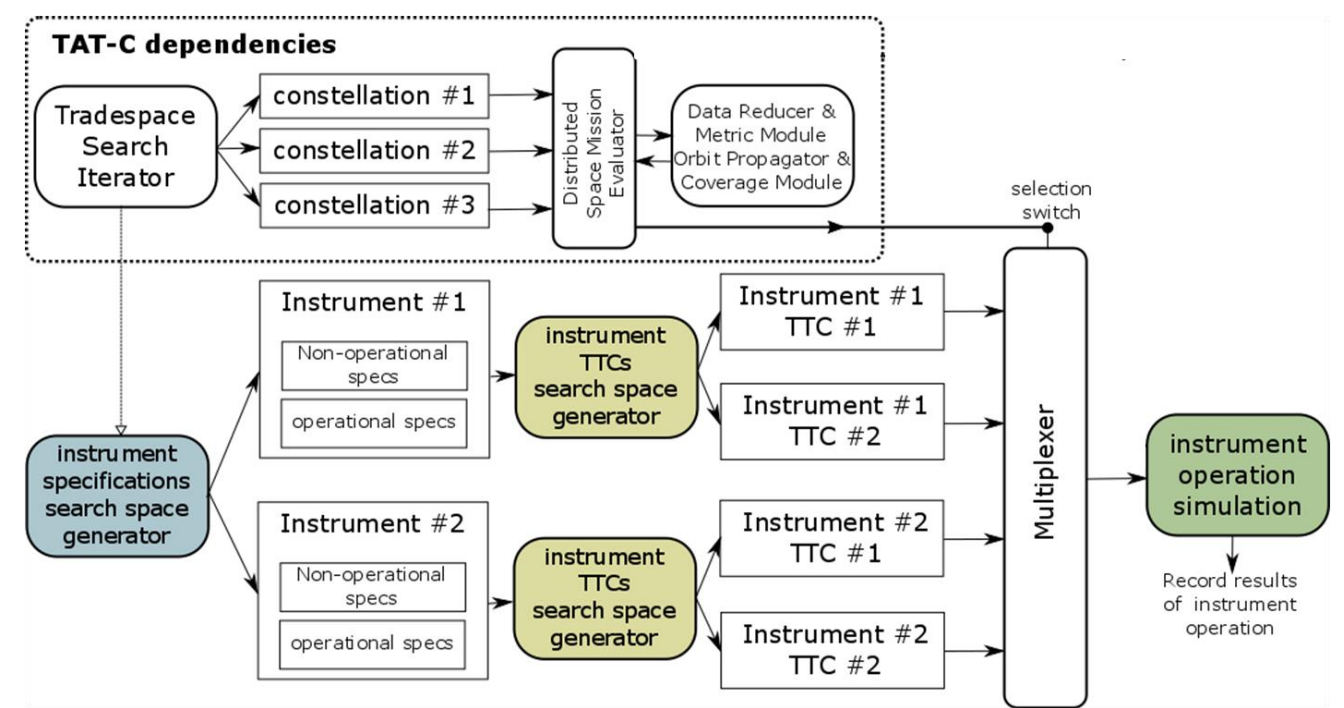

Fig. 1. Architecture of the instrument simulation module. As an example, search space of three constellations, two instruments and two TTCs per instrument is shown. The colored boxes are the three functionalities introduced in the present paper, as the instrument module's submodules.

\section{2) Operational specifications:}

These are specifications of the instrument which can be varied during its operation. For example, the duty cycle of the SAR operation maybe varied within $10 \%$ to $35 \%$ depending on the command issued by the ground-station via Time-Tagged commands (TTCs). Another important specification is the instrument pointing attitude (look-angle in case of SAR).

\section{B. Time-Tagged commands search-space generation sumodule}

Any given instrument is operated according to the commands from the ground-station, uploaded via a TTCs file, covering the simulation mission duration. It can be said that an operating point of the instrument is a sample from the range of operational specifications of the instrument. Further, the command can vary in time during the same mission, thus allowing for potentially infinite number of possibilities of the instrument TTCs.

The search-space generation problem of TTCs is a complicated problem which has not been dealt with in the present work. A simple uniform discretization of all timevarying options will yield an impractically large number of samples to process. Therefore a more sophisticated way of the search-space generation is required. In this work, we assume a nominal and constant set of TTCs for a given instrument.

\section{Instrument operation simulation submodule}

This submodule simulates the operation of the instrument, and is dependent on the instrument capability, input TTCs and satellite position in-orbit, satellite sub-system states such as available power for payload operation. The output of this submodule, is also the output of the entire instrument simulation module, which are the time-varying performance of the instrument and the collected remotely-sensed data.

\section{SAR INSTRUMENT MODELING EXAMPLE}

The instrument module is expected to model a few different concept of operations. For example, Ref [4] describes the tradespace analysis of imagers for constellations, that we will adapt for TAT-C. To demonstrate the trade concept for a representatively different instrument, we provide a radar example in this paper. Table.1 lists the parameters (specifications, and remote-sensed data parameters) of an example instrument, a SAR. The SAR model (side-looking with no-squint) considered is based on the framework developed in [5]. For calculation of swath-width, a spherical Earth model is considered, and is calculated as the length of path along range direction that the antenna half-power beamwidth intersects the ground. The pulse repetition frequency selection framework to ensure unambiguous echo returns is based on the formulation in [6, Section 5] (there appear to be many typographic errors in the quoted reference).

The instrument module uses inputs from the orbit propagator, and is hence dependent on the constellation architecture in general. In Ref [3] four different types of satellite constellation were discussed and trade-offs between them were evaluated in terms of revisit time and deployment strategy using TAT-C. Introduction of the instrument module expands the scope of TAT-C and accommodates additional options and constraints to be taken into consideration while deciding a constellation architecture. Running TAT-C along with predefined user science goals, and available instrument specifications, will output those constellation architectures for which the science goals are fulfilled.

We can also use the instrument simulation module to find out the required specifications of an instrument for a given constellation. For example, in the case of SAR, an important orbit parameter is the altitude of the satellite $h$, which influences the selection of the pulse repetition frequency $f_{p}$, so that echo can be discerned unambiguously. If we desire the end image 
quality (evaluated in terms of resolution $\rho_{a}, \rho_{r}$ and noise equivalent reflectivity $\sigma_{N E Z}$ ) to be above a certain threshold, we may need to vary other operational parameters of the SAR to compensate for change in nominal $f_{p}$.

TABLE I SAR PARAMETERS USED IN INSTRUMENT SIMULATION MODULE. SEE [5] FOR A DETAILED DESCRIPTION. THE NOMINAL VALUES USED FOR TRADESPACE PLOT SHOWN IN FIG. 2, 3, 4 ARE INDICATED IN BRACKETS.

\begin{tabular}{|c|c|c|c|}
\hline \multicolumn{2}{|c|}{ Specifications } & \multirow{2}{*}{$\begin{array}{c}\text { Parameters } \\
\text { from orbit } \\
\text { propagation }\end{array}$} & \multirow{2}{*}{$\begin{array}{c}\text { SAR image } \\
\text { performance } \\
\text { metrics }\end{array}$} \\
\hline Non-operational & Operational & & \\
\hline $\begin{array}{c}\text { Antenna } \\
\text { dimension along } \\
\text { azimuth } D_{a z}(6 \\
\mathrm{m})\end{array}$ & $\begin{array}{c}\text { Peak transmit } \\
\text { power } P_{t} \\
(1 \mathrm{~kW})\end{array}$ & $\begin{array}{c}\text { Altitude } h \\
(500 \mathrm{~km})\end{array}$ & $\begin{array}{c}\text { Noise- } \\
\text { equivalent } \\
\text { reflectivity } \\
\sigma_{N E Z}\end{array}$ \\
\hline $\begin{array}{l}\text { Antenna physical } \\
\text { area } A_{A}\left(12 \mathrm{~m}^{2}\right)\end{array}$ & $\begin{array}{c}\text { Operating } \\
\text { center } \\
\text { wavelength } \lambda \\
(3.12 \mathrm{~cm})\end{array}$ & $\begin{array}{l}\text { velocity of } \\
\text { satellite } v_{x} \\
(7613 \mathrm{~m} / \mathrm{s}))\end{array}$ & $\begin{array}{c}\text { Range } \\
\text { resolution in } \\
\text { ground-plane } \\
\rho_{y}\end{array}$ \\
\hline $\begin{array}{l}\text { Antenna aperture } \\
\text { efficiency } \eta_{a p} \\
(0.5)\end{array}$ & $\begin{array}{l}\text { Pulse width } \\
\tau_{p} \text { (30 us) }\end{array}$ & $\begin{array}{c}\text { Nominal } \\
\text { scene } \\
\text { temperature } T \\
(290 \mathrm{~K})\end{array}$ & $\begin{array}{l}\text { Swath-width } \\
\text { imaged } W_{g r}\end{array}$ \\
\hline $\begin{array}{c}\text { Radar system } \\
\text { losses } L_{\text {radar }}^{d B}(3 \\
\mathrm{dB}) \\
\end{array}$ & $\begin{array}{c}\text { Pulse } \\
\text { repetition } \\
\text { frequency } f_{P}\end{array}$ & \multirow{3}{*}{$\begin{array}{c}\text { Two way } \\
\text { atmospheric } \\
\text { loss (function } \\
\text { of operating } \\
\text { center } \\
\text { frequency) } \\
L_{\text {atmos }}^{d B} \\
(2 \mathrm{~dB})\end{array}$} & $\begin{array}{c}\text { Incidence } \\
\text { angle } \theta_{\text {im }}(45 \\
\text { deg) }\end{array}$ \\
\hline $\begin{array}{c}\text { System noise } \\
\text { factor } F_{N}^{d B}(3 \mathrm{~dB})\end{array}$ & $\begin{array}{c}\text { Signal } \\
\text { bandwidth } B_{T} \\
(100 \mathrm{MHz})\end{array}$ & & \multirow{2}{*}{$\begin{array}{l}\text { Minimum } \\
\text { possible } \\
\text { azimuthal } \\
\text { resolution } \\
\text { (stripmap) } \rho_{a}\end{array}$} \\
\hline $\begin{array}{c}\text { Signal processing } \\
\text { parameters } \\
L_{r}, L_{a}, a_{w r}, a_{w a} \\
\quad \text { all } 1,2) \\
\end{array}$ & $\begin{array}{l}\text { Look-angle to } \\
\text { middle of } \\
\text { swath } \gamma_{m}\end{array}$ & & \\
\hline
\end{tabular}

Fig. 2, 3 and 4 are some tradespace plots of a SAR instrument simulated with low Earth orbit mission with nominal simulated parameters as shown in Table 1. The blank regions correspond to areas where there is either desired echo overlap with the transmit pulse, and/or desired echo overlap with a nadir echo from a previous transmit pulse. Note that $\sigma_{N E Z}$ is inversely proportional to SNR, and hence a lower value indicates better image quality. The operable PRF region is limited, and the PRF specification of SAR can be found from these tradespace plots.

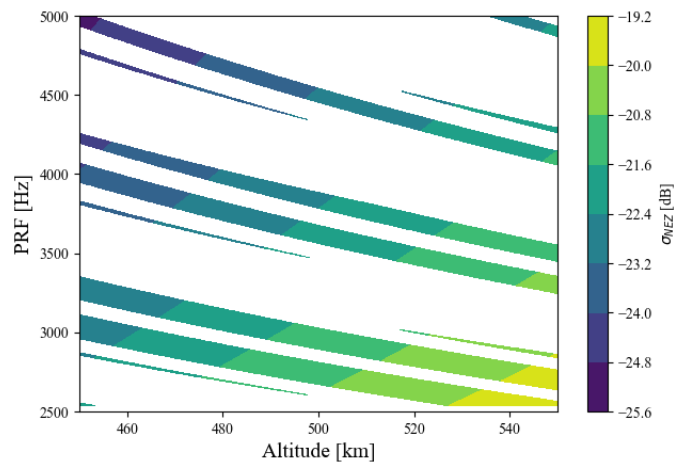

Fig. 2. PRF vs Altitude vs $\sigma_{N E Z}$ tradespace. The minimum PRF is about 2500 $\mathrm{Hz}$ to satisfy to the Nyquist sampling criteria. Altitude uncertainties in mission is expected either due to (1) launch orbit uncertainties (2) gradual decay of altitude during mission lifetime.

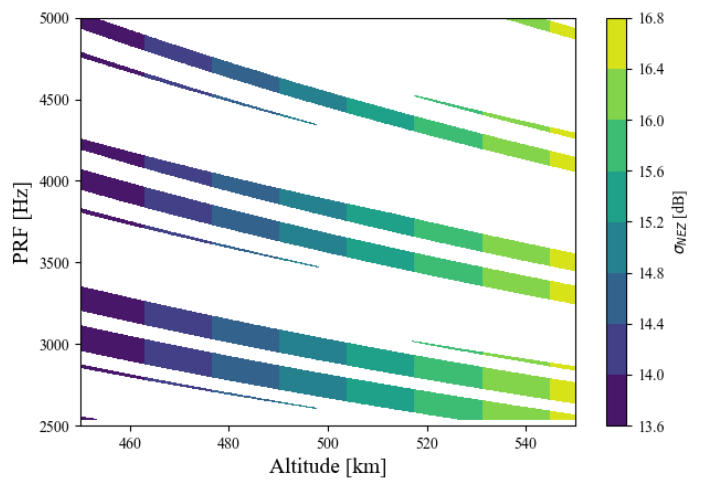

Fig. 3. PRF vs Altitude vs half-power beam illuminated Swath. Swath width increases with increased altitude at the expense of the $\sigma_{N E Z}$. But the later can be compensated by operating at higher PRFs or larger transmit pulse widths.

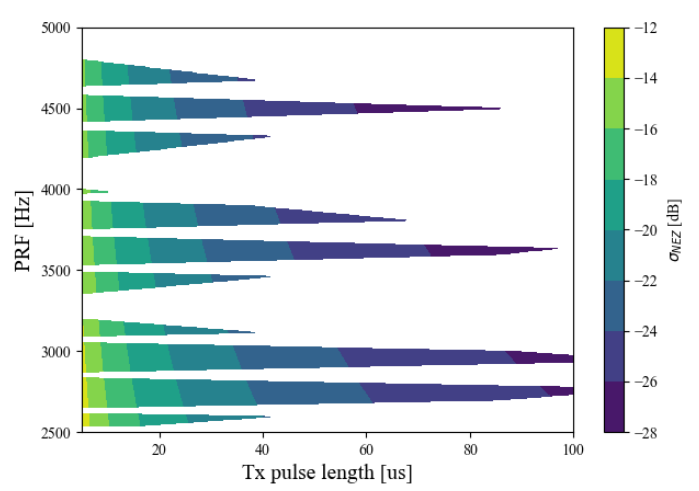

Fig. 4. PRF vs transmit pulse duration vs $\sigma_{N E Z}$. This tradespace plot is drawn for a constant desired swath of $10 \mathrm{~km}$. A larger transmit pulse has fewer regions of operable PRF, but with the advantage being that the $\sigma_{N E Z}$ is improved.

\section{DISCUSSION}

In this paper, we introduce an instrument modeling concept for analysis and design of satellite constellation missions. The instrument module will be developed in Python and integrated with the TAT-C tool by NASA Goddard, which aims to equip satellite constellation mission designers with an open-access tool to analyze constellation tradespaces. The module allows for a more detailed constellation mission analysis involving instrument variables and end-remote sensing data measurement characteristics. An example of a SAR instrument is shown, and various 3D trade-off plots generated. Such plots as generated by the instrument module allows a constellation mission designer to choose proper instrument specifications taking into account operational uncertainties. Conversely, it allows the mission designer to choose proper constellation specifications for a predefined instrument.

In the future, we seek to research more sophisticated ways of search-space generation for the instrument module, especially in case of the TTCs generation. Generation of an optimal TTCs profile allows for adaptive remote sensing in keeping with dynamically changing environments, optimal use of resources for a given constellation and coordination among the satellites for efficient distribution of tasks. 


\section{REFERENCES}

[1] J. LeMoigne et al., "Tradespace Analysis Tool for Designing Constellations (TAT-C)," in IEEE International Geoscience and Remote Sensing Symposium, Fort Worth, TX, 2017.

[2] S. Nag, S. P. Hughes, and J. L. Moigne, "Streamlining the Design Tradespace for Earth Imaging Constellations," in AIAA Space Conference, Long Beach, California, 2016.

[3] S. Nag, S. P. Hughes, and J. LeMoigne, "Navigating the Deployment and Downlink Tradespace for Earth Imaging Constellations," presented at the International Astronautical Congress, Adelaide, Australia, 2017.
[4] S. Nag, T. Hewagama, G. Georgiev, B. Pasquale, S. Aslam, and C. K. Gatebe, "Multispectral Snapshot Imagers onboard Small Satellite Formations for MultiAngular Remote Sensing," IEEE Sens. J., vol. 17, no. 16, pp. 5252-5268, 2017.

[5] A. W. Doerry, "Performance Limits of Synthetic Aperture Radar- Second edition," Sandia Report, Sandia National Laboratories, printed February 2006.

[6] D. L. Bickel, B. C. Brock, and C. T. Allen, "Spaceborne SAR Study: LDRD '92 Final Report," Sandia Report, Sandia National Laboratories, printed March 1993. 